\title{
A Nano-Pharmaceutical Formula of Quercetin Protects from Cardiovascular Complications Associated with Metabolic Syndrome
}

\author{
Osama A. A. Ahmed ${ }^{1,2 *}$, Noura A. Hassan ${ }^{3}$, Ahmad S. Azhar ${ }^{4}$, Mahmoud M. El-Mas ${ }^{5,6}$ and \\ Hany M. El-Bassossy ${ }^{3 *}$ \\ ${ }^{1}$ Department of Pharmaceutics, Faculty of Pharmacy, King Abdulaziz University, Jeddah, Saudi Arabia, ${ }^{2}$ Mohamed Saeed Tamer \\ Chair for Pharmaceutical Industries, King Abdulaziz University, Jeddah, Saudi Arabia, ${ }^{3}$ Department of Pharmacology and \\ Toxicology, Faculty of Pharmacy, Zagazig University, Zagazig, Egypt, ${ }^{4}$ Pediatric Cardiac Center of Excellence, Faculty of Medicine, \\ King Abdulaziz University, Jeddah, Saudi Arabia, ${ }^{5}$ Department of Pharmacology and Toxicology, Faculty of Pharmacy, Alexandria \\ University, Alexandria, Egypt, ${ }^{6}$ Department of Pharmacology and Toxicology, Faculty of Medicine, Kuwait University, Kuwait city, \\ Kuwait
}

OPEN ACCESS

Edited by:

Patrícia Mendonça Rijo,

Universidade Lusófona, Portugal

Reviewed by: Sachin Singh,

Lovely Professional University, India Alptug Karakucuk,

Ankara Medipol University, Turkey

*Correspondence:

Osama A. A. Ahmed oaahmed@kau.edu.sa

Hany M. El-Bassossy

helbassossy@pharmacy.zu.edu.eg

Specialty section: This article was submitted to

Ethnopharmacology,

a section of the journal

Frontiers in Pharmacology

Received: 18 April 2021

Accepted: 19 July 2021

Published: 11 August 2021

Citation:

Ahmed OAA, Hassan NA, Azhar AS,

El-Mas MM and El-Bassossy HM

(2021) A Nano-Pharmaceutical

Formula of Quercetin Protects from

Cardiovascular Complications

Associated with Metabolic Syndrome.

Front. Pharmacol. 12:696981.

doi: 10.3389/fphar.2021.696981
Metabolic syndrome (MetS) is closely associated with the development of cardiovascular diseases. We recently developed a nano-preparation of the flavonoid quercetin (QU) in a selfnanoemulsifying drug delivery system (SNEDDS). The latter comprised a mixture composed of pumpkin seed oil, D- $\alpha$-Tocopherol polyethylene glycol 1,000 succinate and polyethylene glycol. The QU SNEDDS preparations exhibited a considerably higher bioavailability compared with the standard quercetin suspension. Here, we investigated whether the quercetin loaded SNEDDS could offer better protection compared with the standard formulation against cardiovascular complications of MetS in rats. MetS was induced by high fructose, high salt and high fat diet for 12 weeks while the nano-preparation or the standard suspension of quercetin was orally administered for the last 6 weeks. Compared to little effect for the standard quercetin suspension (MQ), the treatment of MetS rats with the quercetin loaded SNEDDS (MNQ) virtually abolished the depressant effect of MetS on contractility index (control, $114 \pm 4$; MetS, $92 \pm 3 ; \mathrm{MQ}, 100 \pm 2 ; \mathrm{MNQ}, 114 \pm 61 / \mathrm{s}$ ) and rate of rise in left ventricular pressure (dP/dtmax) (control, 8,171 $\pm 274 ;$ MetS, 6,664 $\pm 135 ; M Q, 6,776 \pm 108 ; M N Q$, $7,498 \pm 303 \mathrm{mmHg} / \mathrm{s}$ ). Likewise, the prolongation by MetS of electrocardiographic markers of arrhythmogenesis (QTc, JT, and Tpeak-to-Tend intervals) and concomitant rises in dicrotic notch pressure were preferentially reversed by quercetin nano-preparation. On the other hand, the rises in the isovolumic relaxation constant (Tau, denotes diastolic dysfunction), blood pressure, pulse pressure, and difference between systolic and dicrotic pressure (SDP difference) were equally improved by the two preparations of quercetin. Additionally, no differences were noted in the ability of the two quercetin preparations in abrogating the elevated oxidative (MDA) and inflammatory (TNFa) markers in cardiac tissues of MetS rats. Histopathological, microscopical signs of necrosis, inflammatory cell infiltration, and vascular congestion in MetS hearts were more markedly inhibited by the nano-preparation, compared with the standard preparation of quercetin. In conclusion, the quercetin loaded SNEDDS is 


\section{evidently more advantageous than the standard preparation of the drug in alleviating functional} and histopathological manifestations of cardiac damage incited by MetS.

\section{Keywords: quercetin, cardiovascular complications, metabolic syndrome, SNEDDS, nano-formulation}

\section{INTRODUCTION}

Metabolic syndrome (MetS), which is also known as insulin resistance syndrome or syndrome $\mathrm{X}$, is denoted by a group of conditions, namely central obesity, dyslipidemia, hypertension, and hyperglycemia (Alberti et al., 2005). MetS is generally considered injurious due to its linkage with the cardiovascular diseases (CVD) and type 2 diabetes mellitus. Further, a key signal of the related cardiovascular complications is vascular damage and irregular vascular response to vasoconstrictors (facilitation) as well as vasodilators (inhibition) (Bahia et al., 2006). Hypertension, arterial wall stiffness, dyslipidemia, and atherosclerosis are some prominent features of MetS that underlie the provoked vascular damage and dysfunction (Oliver and Webb, 2003; Olijhoek et al., 2004).

The management of MetS relies traditionally on the use of therapies that improve major complications of the disease such as hyperglycemia, hyperlipidemia, and hypertension. Treatment with common medications exerts unpleasant adverse effects such as myalgia, gastritis, hepatitis, hypoglycemia and hypotension in the early stage of treatment (Kaur, 2014). This high level of adverse effects leads to a weak tolerance of the patient especially in the long-term application of the medications (Taghipour et al., 2019). So there is an increasing exploration of using natural products, especially plant polyphenols, along with drugs in order to better regulate the disease (Visioli, 2011). Nanosized drug carriers, consisting of phytochemicals endowed with developed pharmacodynamics and pharmacokinetic characteristics, are a novel therapeutic approach (Taghipour et al., 2019).

Quercetin is a compound present in fruits such as apples and vegetables such as peppers and onions. Quercetin's multiple health benefits have been widely reported for their antiinflammation and antioxidant properties as well as increased endothelium-dependent vasodilation (Murakami et al., 2008; Jagtap et al., 2009; Shen et al., 2012). However, the application of quercetin in pharmaceutical field is limited due to its poor solubility, low bioavailability, poor permeability, and instability (Ahmed et al., 2020). Therefore, it is highly necessary to develop new dosage forms of quercetin with increased solubility and improved bioavailability (Cai et al., 2013). Selfnanoemulsifying drug delivery systems (SNEDDS) have drawn attention for their ability to improve the bioavailability and hence efficacy of orally administered substances like quercetin. SNEDDS systems consist of oil, surfactant, and co-surfactant beside the active ingredient (Yang et al., 2018).

We have previously shown that quercetin improves the compromised vascular reactivity caused by MetS or advanced glycation end-products in vitro (Ahmed et al., 2020). However, the in vivo effect of quercetin administration on different hemodynamic and electrocardiographic parameters was not evaluated. Furthermore, our team has recently developed a TPGS and PSO Based SNEDDS Formulation of quercetin with improved bioavailability and a globule size of $320 \mathrm{~nm}$ and zeta potential of $-28.6 \mathrm{mV}$ (Ahmed et al., 2021). So as a follow-up to our previous reports, the current study tested the hypothesis that the improved bioavailability of the developed SNEDDS preparation of quercetin would prompt better control of cardiovascular complications of MetS. Accordingly, studies were undertaken to assess the possible counterbalancing effects of the SNEDDS pharmaceutical formula of quercetin as compared to the standard formula on hemodynamic, electrocardiographic, left ventricular dynamic, and histopathological derangements in the high fructose/salt rat model of MetS. Further, the research was extended to determine the role of inflammatory, oxidative, and adipokine pathways in the interaction.

\section{METHODS}

\section{Experimental Animals}

For this study, male Wistar rats, 6-8 weeks old, weighing 180-200 g (Zagazig University, Zagazig, Egypt) were used. They were kept in clear polypropylene cages, four rats in each cage, and clean drinking water and rodent pellets were also provided. Under the animal housing conditions, alternating $12 \mathrm{~h}$ of light and dark was employed, at $22 \pm 3^{\circ} \mathrm{C}$ temperature, $50-60 \%$ relative humidity, and sufficient ventilation. The experimental model and animal handling process were followed as per the Ethical Committee for Animal Handling guidelines, Zagazig University (ZU-IACUC/3/F/199/2019).

Animals were randomly divided into five experimental groups. Group 1: control rats (C) that received regular tap water and food pellets for 12 consecutive weeks. Group 2: MetS rats (M) that received fructose (10\%) in drinking water and $\mathrm{NaCl}(3 \%)$ and high-fat diet (25\%) in food pellets for 12 consecutive weeks. Group 3: rats received a single daily dose $(83 \mu \mathrm{mol} / \mathrm{kg})$ of the standard quercetin suspension (MQ) during the last 6 weeks of the study. Group 4: rats received a daily dose of the nano-formula vehicle $(\mathrm{MN})$ in the same volumes of SNEDDS ingredients (Pumpkin seed oil, D- $\alpha$-tocopheryl polyethylene glycol succinate (TPGS), and PEG 200) without quercetin during the last 6 weeks of the study. Group 5: rats received a single daily dose of the quercetin nano-formula preparation (MNQ) at a dose of $83 \mu \mathrm{mol} / \mathrm{kg}$ of quercetin during the last 6 weeks of the study.

\section{Blood Pressure Recording}

For invasive recordings of blood pressure hemodynamics, the procedure described in the existing literature was followed (Azhar and El-Bassossy, 2014; El-Bassossy and Watson, 2015; ElBassossy et al., 2017a; El-Bassossy et al., 2017b). Following the 
administration of anesthesia through a single intraperitoneal injection with $365 \mu \mathrm{mol} / \mathrm{kg}$ ketamine and $45 \mu \mathrm{mol} / \mathrm{kg}$ xylazine, the rats were mounted on regulated heating pads. Further, a microtip pressure transducer (SPR-320; Millar Instruments, Houston, TX, United States) was fitted into the right carotid artery. After $5 \mathrm{~min}$ of stabilization, the signals were noted down for $10 \mathrm{~min}$. The microtip catheter was linked to a PowerLab Data Interface Module on LabChart professional software (version 8.0; AD Instruments, Bella Vista, Australia) containing a blood pressure module on a computer. The BP module was used to quantitatively study the systolic, diastolic, and dicrotic notch blood pressure.

\section{Cardiac Hemodynamics Recording}

After 10 min of recording of blood pressure hemodynamics, the microtip catheter was advanced into the left ventricle under careful pressure control. Then the cardiac hemodynamic signals were recorded for another $10 \mathrm{~min}$. The cardiac hemodynamic module was employed to quantitively determine contractility index, rate of rising in LV pressure (dP/dtmax), isovolumic relaxation constant (Tau), and heart rate (HR).

\section{Electrocardiographic Recording}

The surface ECG was recorded in anaesthetized rats at the same time of cardiac hemodynamic recording by the method described previously by our group (El-Bassossy et al., 2016; El-Bassossy et al., 2017c; El-Bassossy et al., 2018a). The used system was Powerlab (ADI Instruments) connected to a PC running LabChart professional software (version 8.0) containing an ECG module, which quantitatively determines different components of the ECG wave; PR, QTc, and JT intervals.

\section{Biochemical and Immunologic Measurements}

After cardiac hemodynamic recording, a small incision was made in the lower abdomen and $2 \mathrm{ml}$ of blood were withdrawn from the inferior vena cava, allowed to coagulate before separating the serum by centrifugation $\left(8,000 \mathrm{rpm}, 4^{\circ} \mathrm{C}, 10 \mathrm{~min}\right)$. Aliquots of the serum were stored at $-80^{\circ} \mathrm{C}$ for the measurement of insulin and adiponectin levels by rat specific sandwich enzyme-linked immunosorbent assay (ELISA) kits (Crystal Chem inc. Elk Grove Village, IL 60007 United States and MyBioSource, Inc. San Diego, CA, United States respectively) according to manufacturer's guidelines.

Following blood sampling, the heart was excised and $\sim 50 \mathrm{mg}$ from the left ventricle was snap-frozen and stored at $-80^{\circ} \mathrm{C}$ to analyze later for tumor necrosis factor- $\alpha$ (TNF- $\alpha$ ), malondialdehyde (MDA), and protein content using Mybiosource ${ }^{\circledast}$ ELISA rat specific TNF- $\alpha$ assay kit (MyBioSource, Inc. San Diego, CA, United States) and Biodiagnostic kits for MDA and protein (Biodiagnostic ${ }^{\circledR}$, Dokki, Giza, Egypt).

\section{Histopathologic Examination}

The remainder of the heart was placed in $10 \%$ neutral buffered formalin for a duration of 2 days before being moved to $1 \%$ neutral buffered formalin for histopathology (hematoxylin and eosin stain).

\section{Drugs and Chemicals}

The following drugs and chemicals were used in this study: Quercetin (Purity $>95 \%$ by HPLC, Sigma-Aldrich, Dorset, United Kingdom), ketamine ( $>99 \%$. Sigma Pharmaceutical Industries, Menoufia, Egypt), xylazine ( $\geq 99 \%$, Sigma-Aldrich, St. Louis, MO, United States).

\section{Statistical Analysis}

Values are expressed as mean \pm standard error of the mean. Statistical comparisons were carried out using one-way ANOVA, followed by Newman-Keuls' post hoc test using Prism $5{ }^{\circledR}$ software (Graphpad, CA, United States). Probability levels less than 0.05 were considered statistically significant.

\section{RESULTS}

\section{MetS/Quercetin Interaction on Cardiac Hemodynamics}

Feeding rats with a high fructose/salt/fat diet for 12 weeks resulted in marked reductions in cardiac contractility as appearing from the significant decrease in contractility index compared to control animals $(p<0.05$, Figures 1A,D). Daily administration of standard quercetin suspension in a dose of $83 \mu \mathrm{mol} / \mathrm{kg}$ during the last 6 weeks did not significantly affect the reduced cardiac contractility in MetS animals. Administration of the plain nanopharmaceutical formula significantly increased cardiac contractility in MetS but did not completely restore normal cardiac contractility $(p<0.05$, Figures 1A,D). However, the quercetin-loaded nanopharmaceutical formula containing the same dose of quercetin completely restored cardiac contractility to normal levels in control rats $(p<0.05$, Figures 1A,D).

The heart of MetS animals suffered from left ventricular systolic dysfunction as reflected by a significant decrease in the rate of rising in $\mathrm{LV}$ pressure ( $\mathrm{dP} / \mathrm{dtmax}, p<0.05$, Figure $1 \mathrm{~B})$. The treatment with quercetin suspension or the plain nanopharmaceutical formula failed to improve the deteriorated cardiac systolic function in MetS animals. By contrast, the quercetin-loaded nano-pharmaceutical formula almost restored systolic function to its near-control levels ( $p<0.05$, Figure 1B).

The left ventricular diastolic time constant (Tau) was increased in MetS animals compared to control values $(p<0.05$, Figure 1C) indicating impairment in diastolic function. The treatment with any of the three regimens, quercetin suspension, plain nanopharmaceutical formula, or quercetin-loaded nano-pharmaceutical formula, significantly and similarly reversed the rises in Tau provoked by MetS (all at $p<0.05$, Figure 1C).

\section{MetS/Quercetin Electrocardiographic Interaction}

Figure 2 shows that MetS induced by a high fructose/salt/fat diet resulted in delayed ventricular repolarization as reflected by a significant prolongation in QTc, JT, and T peak-Tend intervals compared to control values $(p<0.05)$. The prolonged QTc, JT, and $\mathrm{T}$ peak-Tend intervals were slightly, but not significantly, shortened in MetS rats treated with quercetin suspension or the plain nano-pharmaceutical formula. On the other hand, the 

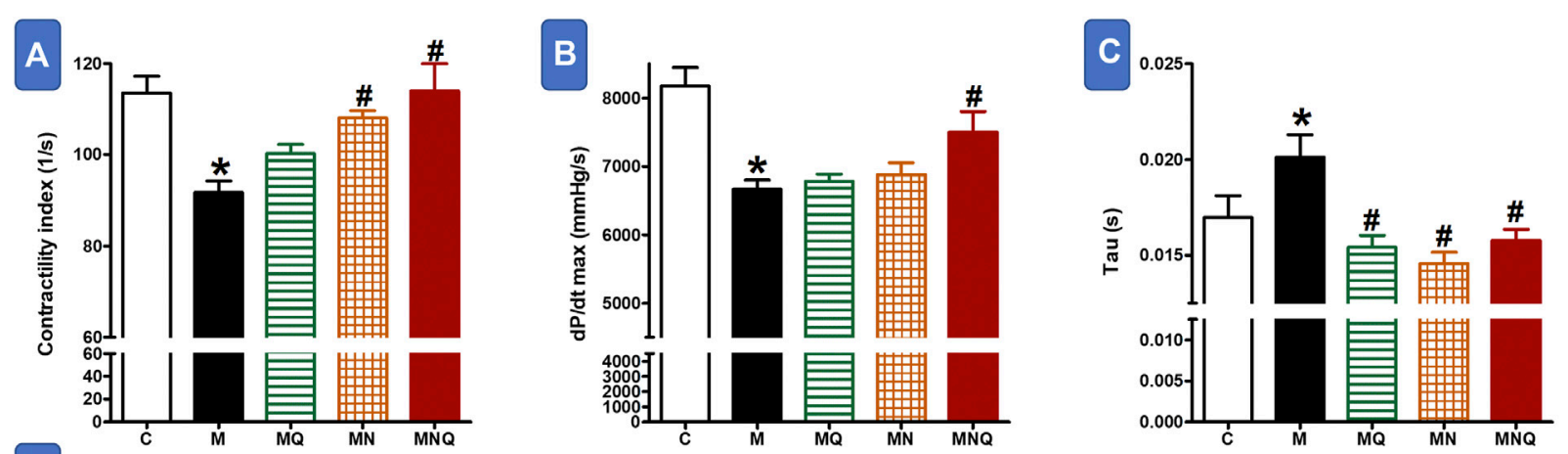

D
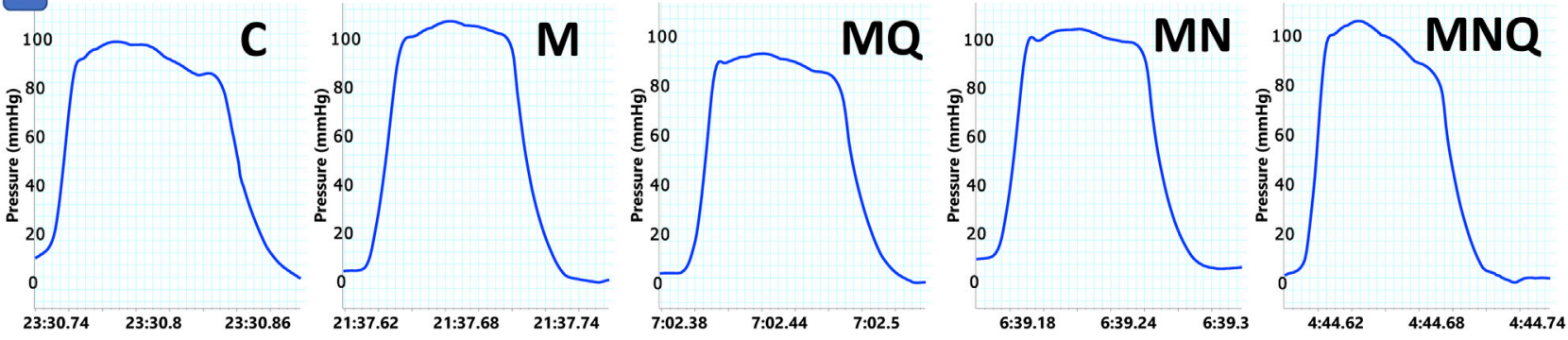

FIGURE 1 | Effect of oral administration of standard quercetin suspension (MQ), plain nano-pharmaceutical formulation (MN) and the quercetin loaded nanopharmaceutical formulation (MNQ) both at $83 \mu \mathrm{mol} / \mathrm{kg} /$ day on contractility index (A), dp/dt max (B) and Tau (C) in metabolic syndrome (M) induced by feeding rats a high fructose (10\% in drinking water), high salt (3\%), high fat (25\%) diet for 12 weeks. Panel D shows representative original recording of ventricular pressure. Results are expressed as mean \pm SEM ( $n=8$ for all groups). ${ }^{*} p<0.05$ when compared to the corresponding control (C) values, $\# p<0.05$ when compared to the corresponding MetS values using one-way ANOVA followed by Dunnet's post-hoc test.

MetS-related prolongation in the duration of these intervals was significantly reduced after treatment with the nano-pharmaceutical formula of quercetin ( $p<0.05$, Figure 2). The quercetin nano formula almost restored normal ventricular repolarization as appearing from the significant decrease in QTc, JT, and T Peak-Tend intervals (Figure 2).

\section{MetS/Quercetin Interaction on Blood Pressure and Heart Rate}

MetS animals showed significant elevations in both systolic blood pressure (SBP) and diastolic blood pressure (DBP) compared to control animals $(p<0.05$, Figures 3A,B,D $)$. Quercetin administration partially reduced the elevations in SBP and DBP compared to MetS animals $(p<0.05)$. The plain nano-formula produced similar reductions in SBP and DBP $(p<0.05)$. However, the nano-pharmaceutical formula of quercetin markedly reduced the elevations in both systolic and diastolic BP nearly to control values $(p<0.05$, Figures 3A,B,D). On the other hand, MetS animals treated with or without any of the three intervening regimens did not produce any change in heart rate compared to the control group (Figure 3C).

\section{MetS/Quercetin Interaction on Pulse Pressure, Dicrotic Notch Pressure, and SDP Difference}

MetS induction resulted in a significant increase in pulse pressure, dicrotic notch pressure, and the difference between systolic and dicrotic pressure (SDP difference, $p<0.05$, Figure 4) compared to the control group. Quercetin administration significantly reduced pulse pressure and SDP difference, but not the dicrotic notch pressure, compared to MetS group (Figure 4). However, daily oral administration of the plain nano-pharmaceutical formulation or the nano-pharmaceutical formula of quercetin in the last 6 weeks significantly reduced pulse pressure, dicrotic notch pressure, and SDP difference compared to MetS rats $(p<0.05$, Figure 4).

\section{MetS/Quercetin Oxidative Interaction}

Induction of MetS in rats caused a significant increase in heart MDA level ( $p<0.05$, Figure 5A) compared to the control group. The treatment with any of the three regimens, quercetin suspension, plain nano-pharmaceutical formula, or quercetin loaded nano-pharmaceutical formula, significantly and similarly reversed the rises in MDA level provoked by MetS $(p<0.05$, Figure 5A).

\section{MetS/Quercetin Inflammatory Interaction}

Induction of MetS in rats caused a significant increase in heart TNFa level $(p<0.05$, Figure 5B) compared to the control group which indicates that MetS is associated with low-grade inflammation. The treatment with any of the three regimens, quercetin suspension, plain nano-pharmaceutical formula, or quercetin loaded nanopharmaceutical formula, significantly reduced the elevated level of TNF- $\alpha$ level caused by MetS ( $p<0.05$, Figure 5B).

Moreover, MetS induction resulted in a significant decrease in serum adiponectin level ( $p<0.05$, Figure 5C) compared to the 

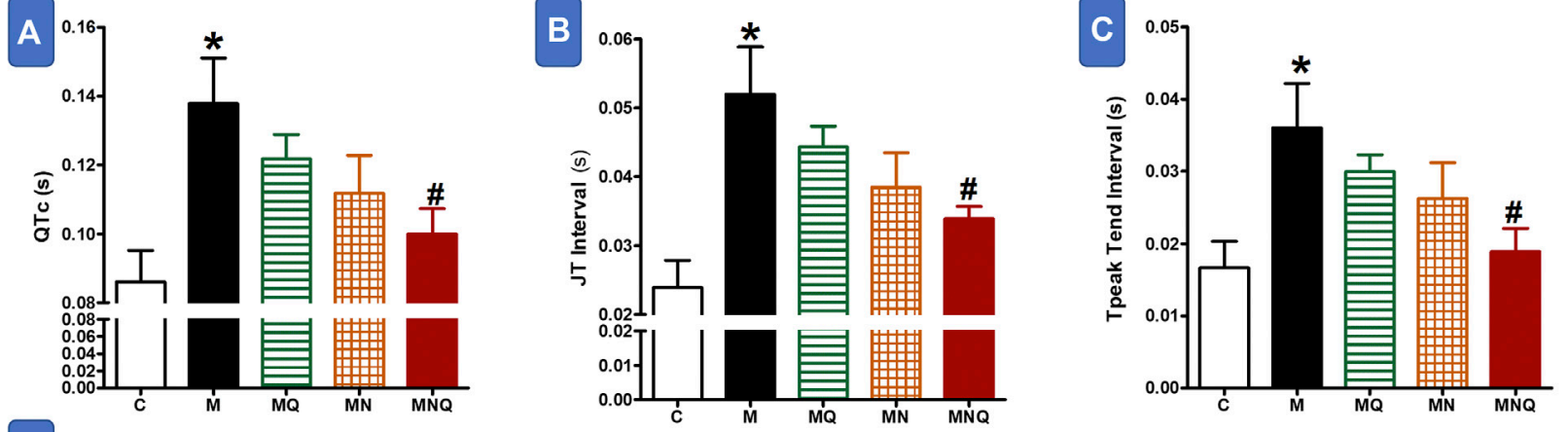

D
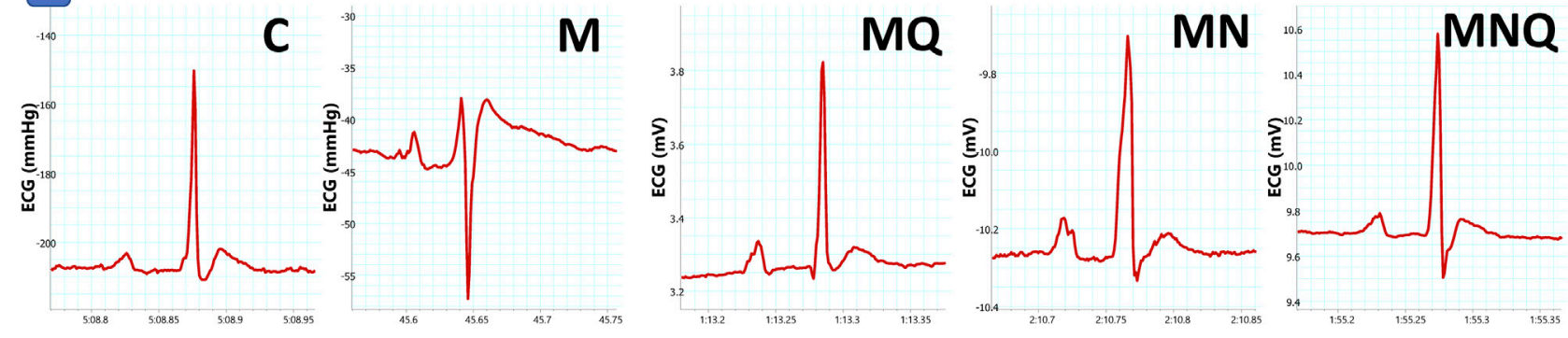

FIGURE 2 | Effect of oral administration of standard quercetin suspension (MQ), plain nano-pharmaceutical formulation (MN) and the quercetin loaded nanopharmaceutical formulation (MNQ) both at $83 \mu \mathrm{mol} / \mathrm{kg} /$ day on QTc (A), JT interval (B) and PR interval (C) in metabolic syndrome (M) induced by feeding rats a high fructose (10\% in drinking water), high salt (3\%) and high fat (25\%) diet for 12 weeks. Panel D shows representative original recording of ECG. Results are expressed as mean \pm SEM ( $n=8$ for all groups). ${ }^{*} p<0.05$ when compared to the corresponding control $(\mathrm{C})$ values, \#p $<0.05$ when compared to the corresponding MetS values using one-way ANOVA followed by Dunnet's post-hoc test.

control group. Daily oral administration of quercetin suspension at a dose of $83 \mu \mathrm{mol} / \mathrm{kg}$ in the last 6 weeks significantly increased serum adiponectin level compared to MetS rats $(p<0.05$, Figure $5 \mathrm{C})$. The plain nano-pharmaceutical formulation increased serum adiponectin level but did not reach the level to be significantly different from MetS. However, the nano-pharmaceutical formula of quercetin did not produce a change in serum adiponectin level compared to the MetS group (Figure 5C).

\section{MetS/Quercetin Interaction on Insulin and Lipid Profile}

Table 1 shows that MetS rats showed significantly elevated serum insulin level compared with the control group. While, quercetin, nano-pharmaceutical formula of quercetin, and even the plain formula, all significantly inhibited the hyperinsulinemia (all at $p<$ $0.05)$. MetS animals showed significant hypertriglyceridemia compared to control $(p<0.05)$ while only the plain formula was able to significantly inhibit this hypertriglyceridemia $(p<$ 0.05). However, neither the MetS animals nor any of the treatments in the current study significantly affected the cholesterol level.

\section{MetS/Quercetin Histopathologic Interaction}

Light microscopy of cardiac myocytes in the control group's ventricles presented a normal histological outcome. Myocytes were found to be branching and cylindrical in shape. An acidophilic sarcoplasm and central oval single nuclei (Figure 6A) were also seen. In MetS hearts, distinct histological changes were noted. Also, disruption and fragmentation of cardiac myocytes were apparent. Most muscle fibers showed cytoplasmic lysis, while numerous myocytes presented dark stained nuclei. The cardiac myocytes were distinguished by wide intercellular spaces. Further, mononuclear cellular infiltration was noticed intermediately among cardiac myocytes (Figures 6B,C; Table 2). The hearts presented partial restoration of the control group's histological pattern in both plain formula (Figure 6D) as well as standard quercetin suspension (Figure 6E). Most cardiac muscle fibers were cylindrical with a central oval-shaped nuclei. However, in between cardiac myocytes, wide intercellular spaces were still noted. Some of the muscle fibers showed striations disappearing. Moreover, little mononuclear cellular infiltration could be noted in between some of the cardiac myocytes (Table 2). In the NQ group, the histological pattern was similar to that of the control group. Most cardiac muscle fibers were found to be cylindrical with a central oval-shaped nuclei. Moderately wide intercellular gaps were found between cardiac myocytes (Figure 6F; Table 2).

\section{DISCUSSION}

The current study clearly shows that the provided SNEDDS preparation significantly potentiates quercetin cardioprotective 

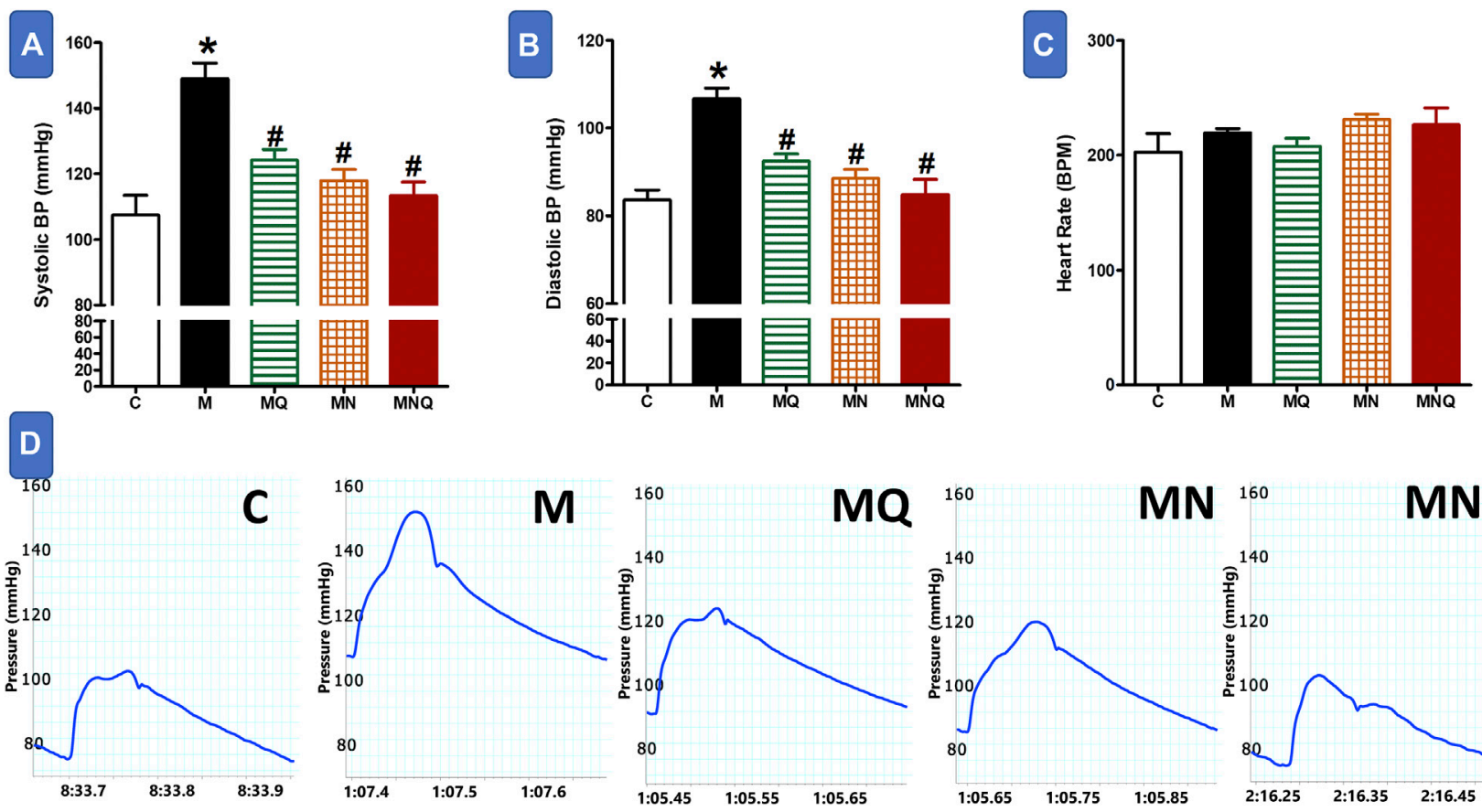

FIGURE 3 | Effect of oral administration of standard quercetin suspension (MQ), plain nano-pharmaceutical formulation (MN) and the quercetin loaded nanopharmaceutical formulation (MNQ) both at $83 \mu \mathrm{mol} / \mathrm{kg} /$ day on systolic BP (A), diastolic BP (B) and heart rate (C) in metabolic syndrome (M) induced by feeding rats a high fructose (10\% in drinking water), high salt (3\%) and high fat (25\%) diet for 12 weeks. Panel D shows representative original recording of arterial hemodynamics. Results are expressed as mean \pm SEM ( $n=8$ for all groups). ${ }^{*} p<0.05$ when compared to the corresponding control (C) values, \#p $<0.05$ when compared to the corresponding MetS values using one-way ANOVA followed by Dunnet's post-hoc test.

effect. While the standard quercetin suspension failed to completely protect from cardiovascular dysfunction in metabolic syndrome, the current nano-formula containing the same dose of quercetin almost completely restored normal cardiovascular function.

To study the efficacy of the current SNEDDs of quercetin on the cardiovascular function we tested its effect on left ventricular pressure measurements, ECG, and arterial hemodynamics and compared the resulted effect with that of quercetin suspension and the plain nano-formulation. Here, we evaluated the effect of the three regimens on cardiac contractility due to its importance in safety assessment studies since either an increase or a decrease may be harmful under certain clinical situations (Sarazan et al., 2012). MetS showed signs of impaired cardiac contractility as evidenced by the reduction in parameters like maximal left ventricular pressure $(\mathrm{dP} / \mathrm{dt} \max )$ and contractility index which can exacerbate the consequences of clinical heart failure (Sarazan et al., 2012). Quercetin suspension did not improve dP/dt max or cardiac contractility index, while nano-formulation containing the same dose of quercetin succeeded in restoring normal cardiac contractility. We cannot ignore the contribution of the plain nano-formulation in the previous effect as its administration alone without quercetin significantly increased cardiac contractility to some extent.

To calculate the diastolic function, the left ventricular diastolic time constant ( $\mathrm{Tau}$ ) was measured which was significantly prolonged in MetS animals. The nano-pharmaceutical formula of quercetin normalized Tau and its effect is similar to that of quercetin suspension or the plain nano-pharmaceutical formulation.

Notably, each MetS component such as dyslipidemia, elevated blood pressure, insulin resistance, and abdominal obesity, is recognized to damage the cardiac structure induce arrhythmias. Therefore, the coexistence of MetS and ECG deviation can possibly mean more risk of death due to sudden cardiac arrest (Abiodun et al., 2019). ECG of MetS animals revealed a significant prolongation of QTc, JT, and T peakTend intervals compared to control. On the other hand, the nano-formulation of quercetin augmented the effect of quercetin on ECG vectors reflecting ventricular activity which cannot be achieved by quercetin suspension alone.

Besides studying the effect of nano-formulation of quercetin on the electrical contraction of the heart, we also studied its effect on arterial waveform signal which reflects mechanical contraction of the heart. In the present work, MetS was associated with increased systolic and diastolic blood pressure but normal heart rate which is consistent with our previous study (Inanir et al., 2020). Oral administration of nano-formulation of quercetin reduced both systolic and diastolic blood pressure without affecting heart rate. Both quercetin suspension and the plain nano-pharmaceutical formulation reduced the elevated systolic and diastolic blood pressure but the effect of nano-formulation of quercetin on blood pressure is more prominent than their effects. 


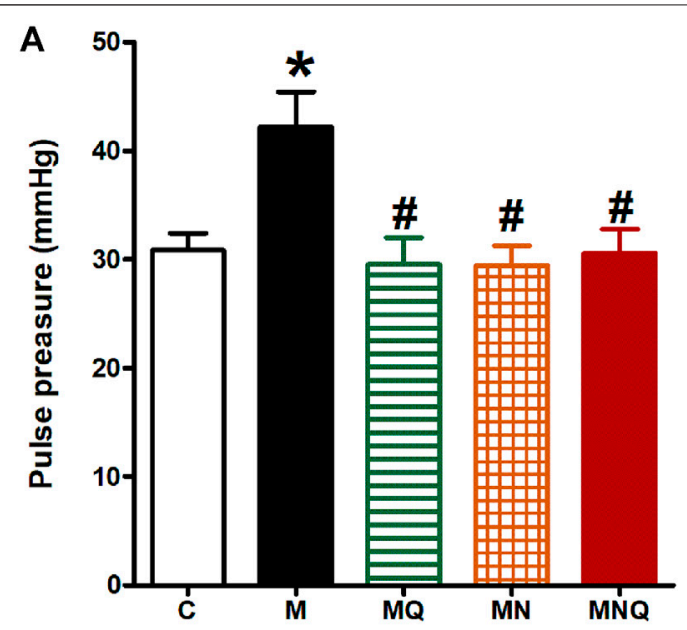

B

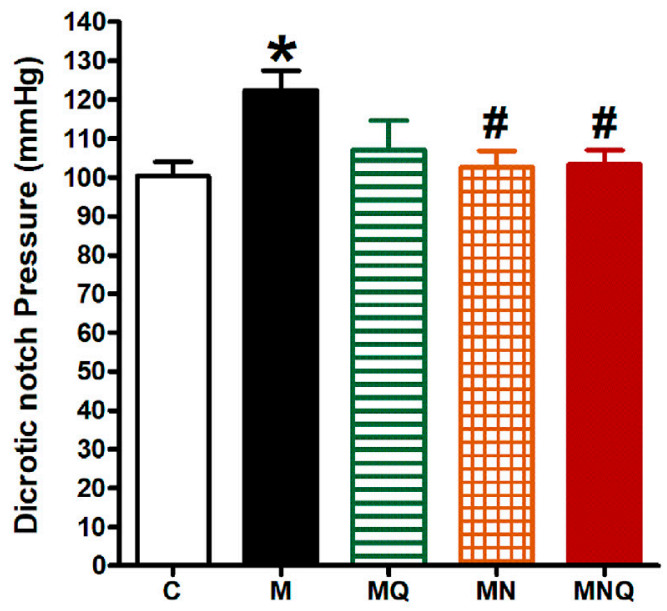

C

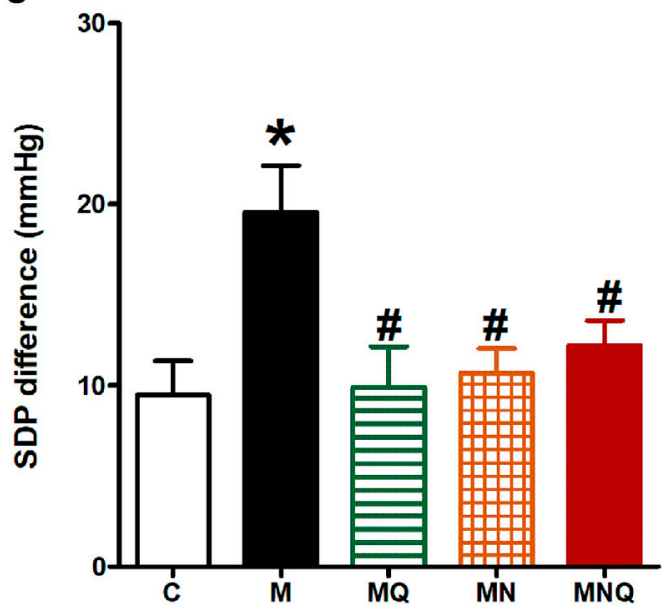

FIGURE 4 | Effect of oral administration of standard quercetin suspension (MQ), plain nano-pharmaceutical formulation (MN) and the quercetin loaded nano-pharmaceutical formulation (MNQ) both at $83 \mu \mathrm{mol} / \mathrm{kg} /$ day on pulse pressure (A), dicrotic notch pressure (B) and SDP difference $\mathbf{( C )}$ in metabolic syndrome $(\mathbf{M})$ induced by feeding rats a high

(Continued)
FIGURE 4 | fructose (10\% in drinking water), high salt (3\%) and high fat (25\%) diet for 12 weeks. Results are expressed as mean $\pm \operatorname{SEM}(n=8$ for all groups). ${ }^{*} p<0.05$ when compared to the corresponding control $(\mathrm{C})$ values, $\# p<0.05$ when compared to the corresponding MetS values using one-way ANOVA followed by Dunnet's post-hoc test.

Moreover, three more parameters of arterial hemodynamics were evaluated in our present study; 1) pulse pressure, 2) the dicrotic notch pressure, and 3) the SDP difference. MetS was associated with increased pulse pressure, dicrotic notch pressure, and SDP difference, which is in accordance with our previous work (Azhar and El-Bassossy, 2015). The impairment in arterial hemodynamics observed in the present study increases the risk for cardiovascular diseases due to increased arterial stiffness and impaired cardiovascular performance and efficiency. The plain nano-pharmaceutical formulation or the nano-pharmaceutical formula of quercetin restored normal arterial hemodynamic measurements to that of control. While quercetin suspension improved pulse pressure and SDP difference but lacked a significant effect on dicrotic notch pressure.

Further, it is known that adipocytes produce signaling molecules, which are called adipokines or adipocytokines and influence insulin action, including adiponectin and TNF- $\alpha$ (Pittas et al., 2004). In the current study, the serum adiponectin level in MetS animals was noted to be considerably lower than that in the control group. Low plasma adiponectin levels are also usually found in many states that are generally linked to insulin resistance, such as cardiovascular disease (Kumada et al., 2003) and hypertension (Adamczak et al., 2003). Moreover, in the current study, MetS animals were distinguishable by their low-grade inflammation, as seen in the considerably higher TNFa serum level. Notably, elevated levels were earlier noted in the inflammatory cytokine TNF- $\alpha$ in MetS animal models which were highly correlated with vascular complications (El-Bassossy et al., 2011; Mahmoud et al., 2012; Mahmoud et al., 2013).

The rise in cardiac TNFa caused by MetS was similarly eliminated by the three regimens but the reduction in adiponectin caused by MetS was eliminated only by quercetin suspension. The anti-inflammatory effect of quercetin in metabolic syndrome was previously demonstrated through its effect on TNF- $\alpha$ and adiponectin (Rivera et al., 2008; Wein et al., 2010; Zhang et al., 2018) and the antiinflammatory effect of the PSO was also demonstrated in previous studies (Bardaa et al., 2016; Bardaa et al., 2020).

Finally, oxidative stress was also noted to contribute significantly in endothelial dysfunction and coronary artery disease (Heitzer et al., 2001). In the present study, the MDA level in MetS animals increased significantly compared to control animals which is in accordance with our previous study (Hassan et al., 2018). The nano-pharmaceutical formula of quercetin antioxidant activity was almost near that of the plain nanopharmaceutical formulation and quercetin suspension. This confirms the anti-oxidant activity of the plain nanopharmaceutical formulation which can be due to the presence of D-a-tocopheryl polyethylene glycol succinate (Engin, 2009). These results proved that the components of the plain nanopharmaceutical formulation besides possessing antioxidant and 


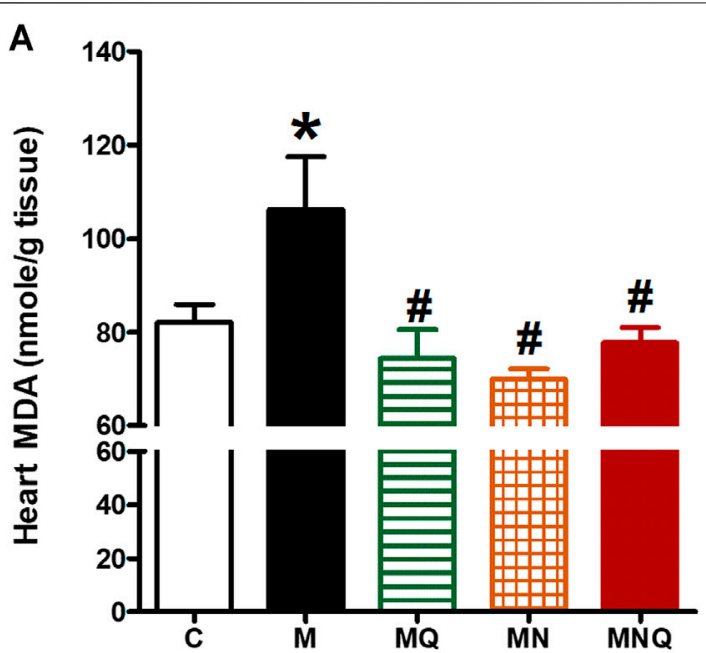

B

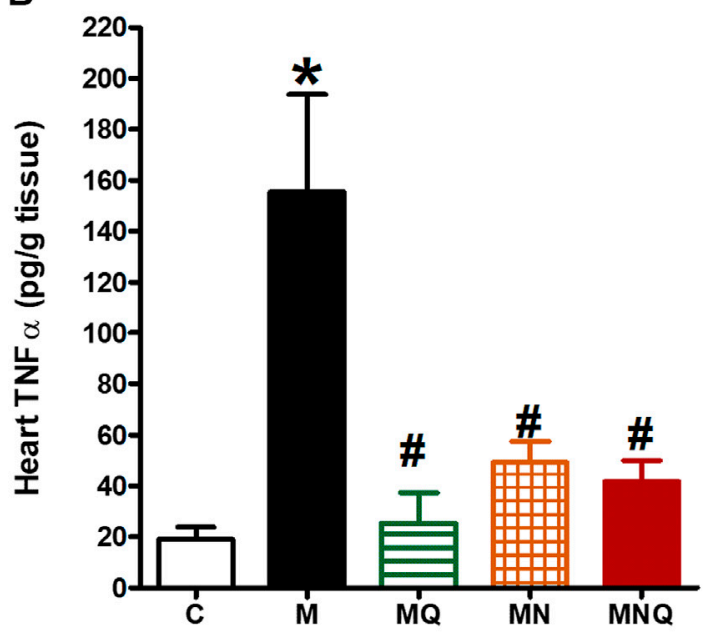

C

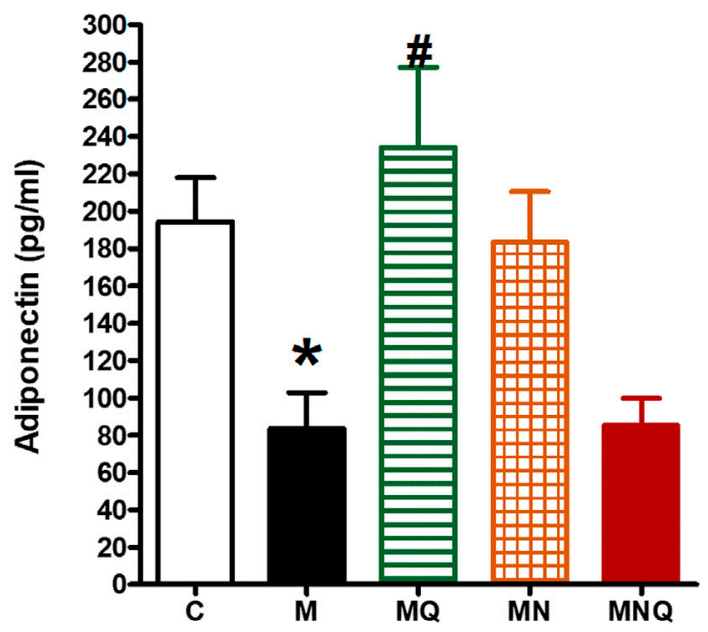

FIGURE 5 | Effect of oral administration of standard quercetin suspension (MQ), plain nano-pharmaceutical formulation (MN) and the quercetin loaded nano-pharmaceutical formulation (MNQ) both at $83 \mu \mathrm{mol} / \mathrm{kg} /$ day on heart MDA (A), heart TNF- $\alpha(\mathbf{B})$ and serum adiponectin $(\mathbf{C})$ in metabolic syndrome $(\mathrm{M})$ induced by feeding rats a high
FIGURE 5 | fructose (10\% in drinking water), high salt (3\%) and high fat (25\%) diet for 12 weeks. Results are expressed as mean \pm SEM ( $n=8$ for all groups). ${ }^{*} p<0.05$ when compared to the corresponding control $(\mathrm{C})$ values, $\# p<0.05$ when compared to the corresponding MetS values using one-way ANOVA followed by Dunnet's post-hoc test.
TABLE 1 | Effect of oral administration of standard quercetin suspension (MQ) plain nano-pharmaceutical formulation (MN) and the quercetin loaded nanopharmaceutical formulation (MNQ) both at $83 \mu \mathrm{mol} / \mathrm{kg} /$ day on serum insulin, triglycerides (TG) and total cholesterol (TC) levels, in metabolic syndrome (M) induced by feeding rats a high fructose (10\% in drinking water), high salt (3\%), high fat (25\%) diet for 12 weeks.

\begin{tabular}{lccc} 
Groups & Insulin $\mathbf{( n g / m l )}$ & TG $(\mathbf{m g} / \mathbf{d l})$ & TC $(\mathbf{m g} / \mathbf{d l})$ \\
\hline $\mathrm{C}$ & $1.14 \pm 0.22$ & $77.5 \pm 5.31$ & $114.1 \pm 8.03$ \\
$\mathrm{M}$ & $3.31^{\mathrm{a}} \pm 0.61$ & $162.8^{\mathrm{a}} \pm 21.08$ & $110.0 \pm 6.26$ \\
$\mathrm{MQ}$ & $1.79^{\mathrm{b}} \pm 0.47$ & $170.8 \pm 25.75$ & $114.3 \pm 7.34$ \\
$\mathrm{MN}$ & $1.71^{\mathrm{b}} \pm 0.23$ & $92.43^{\mathrm{b}} \pm 15.29$ & $98.1 \pm 10.45$ \\
$\mathrm{MNQ}$ & $1.78^{\mathrm{b}} \pm 0.33$ & $133.10 \pm 13.90$ & $130.5 \pm 9.42$
\end{tabular}

Results are expressed as mean \pm SEM ( $n=8$ for all groups).

${ }^{a} p<0.05$ when compared to the corresponding control values.

${ }^{b} \mathrm{p}<0.05$ when compared to the corresponding MetS values using one-way ANOVA

followed by Dunnet's post-hoc test.

anti-inflammatory activity, keep and maintain the antioxidant and the anti-inflammatory properties of quercetin itself and suggest its possible role in combating cardiovascular diseases.

The effect of the nano-pharmaceutical formula of quercetin on circulating insulin level and lipid profile, two of the main components of MetS, was also investigated in our present work. Our findings showed that the MetS was characterized by hyperinsulinemia and hypertriglyceridemia but normal cholesterol level. Hyperinsulinemia was eliminated similarly by the three regimens, but hypertriglyceridemia was reduced only by the plain nano-pharmaceutical formulation. These observations strongly suggest that the cardiovascular-protective effect exerted by SNEDDs of quercetin in part related to the normalization of circulating insulin level but still the main role in its cardiovascular protection is due to enhancing the bioavailability of quercetin-loaded SNEDDs preparation obvious from its prominent effect on different cardiovascular physiological indices previously discussed in the manuscript.

This is further explained by the left ventricle histological examination. MetS showed cardiac muscle fiber degeneration, myocardial necrosis, and inflammation which partially improved after the administration of quercetin suspension or the plain nanopharmaceutical formulation. Noteworthy that the improvement in cardiac architecture of animals received plain nano-pharmaceutical formulation is more than that of quercetin suspension due to the reduction in the inflammatory cells infiltration and absence of necrotic tissue. The more improvement in heart tissue seen with the plain nano-pharmaceutical formulation is related to the antioxidant and anti-inflammatory components of the formulation which due to their combination in SNEDDs exhibited better bioavailability than quercetin suspension itself. However, the nano-pharmaceutical formula of quercetin succeeded in restoring the heart tissue pattern nearly similar to 

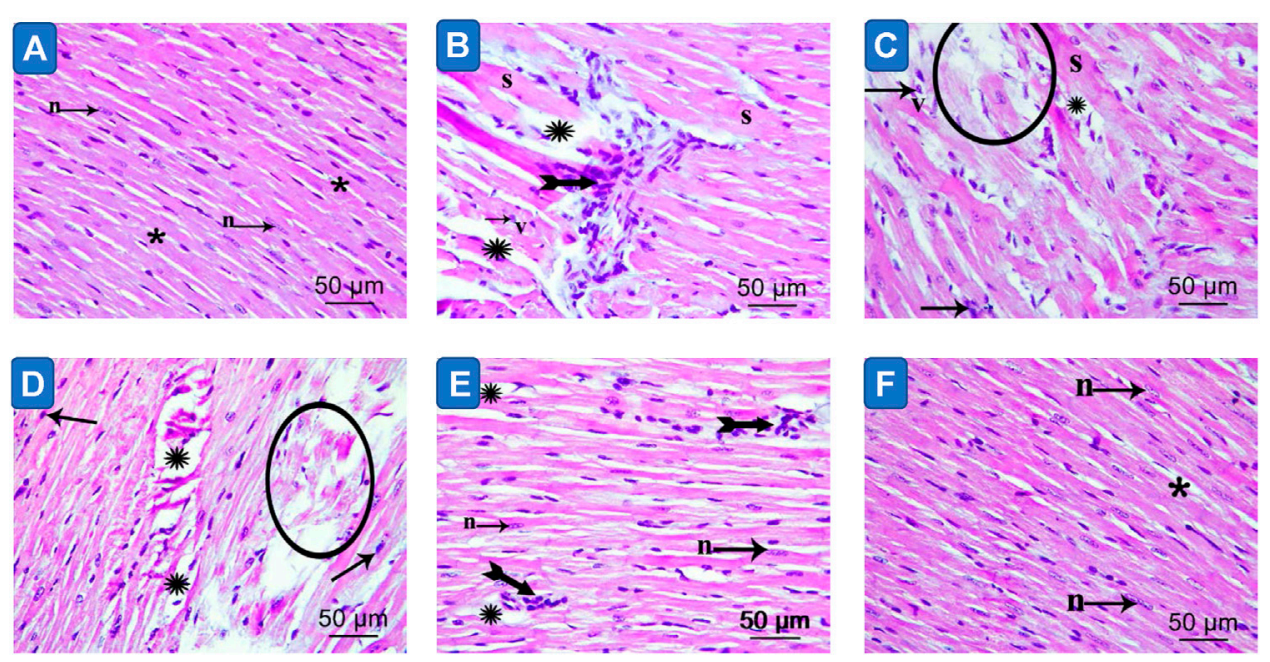

FIGURE 6 | Photomicrograph of sections in the ventricular wall of control (image A), Metabolic syndrome (images B, C), plain formula (image D), quercetin suspension (image E) and quercetin nano-formula (image F) groups. Bifid arrows represent; mononuclear cellular infiltration was evident in between the cardiac myocytes. Circle represent degenerated muscle fibers. "S" represents cardiomyocyte showing loss of striation. Small arrow represents myocytes exhibit dark stained nuclei. "v" represents monocytes with vacuolated cytoplasm (v) Scale bar $=50 \mu \mathrm{m}, \times 400$.

TABLE 2 |Scoring of histopathological findings of hearts of control (C), metabolic syndrome (M), plain nano-pharmaceutical formulation (MN), standard quercetin suspension (MQ) and the quercetin loaded nano-pharmaceutical formulation (MNQ) groups.

\begin{tabular}{lcccc} 
Groups & Myocardial necrosis & Myocardial fibrosis & $\begin{array}{c}\text { Myocarditis (inflammatory cells } \\
\text { infiltrations) }\end{array}$ & $\begin{array}{c}\text { Congested of intramuscular } \\
\text { blood vessels }\end{array}$ \\
\hline$C$ & -- & -- & -- & -- \\
$M$ & +++ & -- & +++ & ++ \\
$M Q$ & ++ & -- & ++ & ++ \\
$M N$ & -- & -- & -- & + \\
$M N Q$ & -- & -- & --
\end{tabular}

Semi-quantitative lesions score system was designed as follows ( $0=$ no alterations, $+=$ mild alterations $(15-25 \%),++=$ moderate alterations (35-65\%), $+++=$ severe alterations (up to $70 \%$ ).

that of the control group, which proved the synergistic effect produced by both enhancing the bioavailability of quercetin and the beneficial effect of the SNEDDs components.

To the best of our knowledge, no report has utilized the combination of PSO (oil), TPGS (emulsifier), and PEG 200 (co-surfactant) as a promising carrier system for the improved delivery and synergistic efficacy of quercetin for protection from cardiovascular complications associated with MetS. The nanopharmaceutical formula of quercetin consists of safe ingredients forming a nano-emulsion. PSO utilized is a natural oil and TPGS and PEG are approved by FDA. All ingredients of the formulation have a reported antioxidant activity. TPGS, the water-soluble derivative of natural vitamin $\mathrm{E}$, has an amphiphilic excellent solubilizer, emulsifier, permeation, and bioavailability enhancer of hydrophobic drugs (Zhang et al., 2012; Guo et al., 2013; Yang et al., 2018). TPGS has a P-glycoprotein (P-gp) inhibition activity that could augment, with solubilizing ability, the efficacy of quercetin (Dintaman and Silverman, 1999).

Our recent study on the pharmacokinetic properties of quercetin-loaded SNEDDs preparation, showed 149.8\% improvement in bioavailability of quercetin in SNEDDS relative to its suspension (Ahmed et al., 2021). The improvement in pharmacokinetic results of the quercetinloaded SNEDDs formula could be a result of SNEDDS ability to enhance the permeability of the gut membrane for the transport of oily compounds (Cho et al., 2014). Furthermore, the instant self-emulsification offers quercetin in small globule solubilized form that massively increases the surface area for quercetin absorption in the gastrointestinal tract (Bu et al., 2014).

Taking into consideration that the enhanced effect of quercetin due to the current nano-formulation is not only due to enhanced bioavailability due to SNEDDs formation as the plain nanoformulation (with no quercetin) showed a marked cardioprotective effect. This points to a direct effect of the well-selected SNEDDS ingredients, Pumpkin seed oil, D- $a$-tocopheryl polyethylene glycol succinate (TPGS), and PEG 200, in cardiovascular protection. Pumpkin oil was chosen over other oils in the current SNEDDS preparation because of its anti-inflammatory effect (Fahim et al., 1995) while D- $\alpha$-tocopheryl polyethylene glycol succinate was chosen based on its antioxidant activities (Engin, 2009). Several reports from our laboratories and others showed key roles of inflammation and oxidative stress in cardiovascular function associated with 
metabolic syndrome (El-Bassossy et al., 2009; El-Bassossy and Watson, 2015; El-Bassossy et al., 2018b).

In conclusion, the quercetin loaded SNEDDS is obviously more advantageous than the standard preparation of the drug in alleviating left ventricular and electro-mechanical cardiac dysfunction associated with MetS and in improving histopathological manifestations of cardiac damage incited by MetS. This beneficial effect is mediated through improving quercetin bioavailability supported by the well-selected SNEDDS ingredients, Pumpkin seed oil, D-a-tocopheryl polyethylene glycol succinate (TPGS) and PEG 200 which themselves showed marked cardioprotective effect.

\section{DATA AVAILABILITY STATEMENT}

The raw data supporting the conclusion of this article will be made available by the authors, without undue reservation.

\section{ETHICS STATEMENT}

The animal study was reviewed and approved by The experimental design and animal handling procedures were

\section{REFERENCES}

Abiodun, A., Oladimeji, A., Bamidele, T., Adewole, A., and Mayowa, O. (2019). Prevalence of ECG Abnormalities Among Adults with Metabolic Syndrome in a Nigerian Teaching Hospital. Afr. Health Sci. 19, 2829-2838. doi:10.4314/ ahs.v19i4.4

Adamczak, M., Wiecek, A., Funahashi, T., Chudek, J., Kokot, F., and Matsuzawa, Y. (2003). Decreased Plasma Adiponectin Concentration in Patients with Essential Hypertension. Am. J. Hypertens. 16, 72-75. doi:10.1016/s0895-7061(02) 03197-7

Ahmed, O. A. A., Azhar, A. S., Tarkhan, M. M., Balamash, K. S., and El-Bassossy, H. M. (2020). Antiglycation Activities and Common Mechanisms Mediating Vasculoprotective Effect of Quercetin and Chrysin in Metabolic Syndrome. Evid. Based Complement. Alternat Med. 2020, 3439624. doi:10.1155/2020/3439624

Ahmed, O. A. A., El-Bassossy, H. M., El-Sayed, H. M., and El-Hay, S. S. A. (2021). Rp-HPLC Determination of Quercetin in a Novel D- $\alpha$-Tocopherol Polyethylene Glycol 1000 Succinate Based SNEDDS Formulation: Pharmacokinetics in Rat Plasma. Molecules 26, 1435. doi:10.3390/ molecules 26051435

Alberti, K. G., Zimmet, P., Shaw, J., and Group, I. D. F. E. T. F. C. (2005). The Metabolic Syndrome-Aa New Worldwide Definition. Lancet 366, 1059-1062. doi:10.1016/S0140-6736(05)67402-8

Azhar, A., and El-Bassossy, H. M. (2014). Pentoxifylline Alleviates Cardiac Ischemia and Dysfunction Following Experimental Angina in Insulin Resistance. PLoS One 9, e98281. doi:10.1371/journal.pone.0098281

Azhar, A., and El-Bassossy, H. M. (2015). Pentoxifylline Alleviates Hypertension in Metabolic Syndrome: Effect on Low-Grade Inflammation and Angiotensin System. J. Endocrinol. Invest. 38, 437-445. doi:10.1007/s40618-014-0209-z

Bahia, L., Aguiar, L. G., Villela, N., Bottino, D., Godoy-Matos, A. F., Geloneze, B., et al. (2006). Relationship between Adipokines, Inflammation, and Vascular Reactivity in Lean Controls and Obese Subjects with Metabolic Syndrome. Clinics (Sao Paulo) 61, 433-440. doi:10.1590/s1807-59322006000500010

Bardaa, S., Ben Halima, N., Aloui, F., Ben Mansour, R., Jabeur, H., Bouaziz, M., et al. (2016). Oil from Pumpkin (Cucurbita Pepo L.) Seeds: Evaluation of its Functional Properties on Wound Healing in Rats. Lipids Health Dis. 15, 73-12. doi:10.1186/s12944-016-0237-0 as indicated by the guidelines of the Ethical Committee for Animal Handling at Zagazig University (ZU-IACUC/3/F/ 199/2019).

\section{AUTHOR CONTRIBUTIONS}

Conceptualization, OA, HE-B, ME-M; methodology, NH, AA; software, HE-B; validation, AA, ME-M, and $\mathrm{NH}$ formal analysis, $\mathrm{NH}, \mathrm{HE}-\mathrm{B}$, and $\mathrm{ME}-\mathrm{M}$; investigation, $\mathrm{NH}$; resources, $\mathrm{OA}$; data curation, $\mathrm{HE}-\mathrm{B}$ and $\mathrm{NH}$; writingoriginal draft preparation, $\mathrm{HE}-\mathrm{B}, \mathrm{AA}$ and $\mathrm{NH}$; writingreview and editing, OA, HE-B, ME-M; visualization, AA, $\mathrm{ME}-\mathrm{M}$, and HE-B; supervision, $\mathrm{OA}$ and $\mathrm{HE}-\mathrm{B}$; project administration, OA; funding acquisition, OA.

\section{FUNDING}

This work was supported by the National Science, Technology and Innovation Plan (NSTIP) strategic technologies program in the Kingdom of Saudi Arabia, Project No. 14-BIO929-03. The authors acknowledge the technical support of Science and Technology unit, King Abdulaziz University.

Bardaa, S., Turki, M., Ben Khedir, S., Mzid, M., Rebai, T., Ayadi, F., et al. (2020). The Effect of Prickly Pear, Pumpkin, and Linseed Oils on Biological Mediators of Acute Inflammation and Oxidative Stress Markers. Biomed. Res. Int. 2020, 5643465. doi:10.1155/2020/5643465

Bu, H., He, X., Zhang, Z., Yin, Q., Yu, H., and Li, Y. (2014). A TPGS-Incorporating Nanoemulsion of Paclitaxel Circumvents Drug Resistance in Breast Cancer. Int. J. Pharm. 471, 206-213. doi:10.1016/j.ijpharm.2014.05.039

Cai, X., Fang, Z., Dou, J., Yu, A., and Zhai, G. (2013). Bioavailability of Quercetin: Problems and Promises. Curr. Med. Chem. 20, 2572-2582. doi:10.2174/ 09298673113209990120

Cho, Y. H., Lee, S. Y., Jeong, D. W., Choi, E. J., Kim, Y. J., Lee, J. G., et al. (2014). Effect of Pumpkin Seed Oil on Hair Growth in Men with Androgenetic Alopecia: a Randomized, Double-Blind, PlaceboControlled Trial. Evid. Based Complement. Alternat Med. 2014, 549721. doi:10.1155/2014/549721

Dintaman, J. M., and Silverman, J. A. (1999). Inhibition of P-Glycoprotein by D-Alpha-Tocopheryl Polyethylene Glycol 1000 Succinate (TPGS). Pharm. Res. 16, 1550-1556. doi:10.1023/a:1015000503629

El-Bassossy, H. M., Al-Thubiani, W. S., Elberry, A. A., Mujallid, M. I., Ghareib, S. A., Azhar, A. S., et al. (2017c). Zingerone Alleviates the Delayed Ventricular Repolarization and AV Conduction in Diabetes: Effect on Cardiac Fibrosis and Inflammation. PLoS One 12, e0189074. doi:10.1371/journal.pone.0189074

El-Bassossy, H. M., Awan, Z., and El-Mas, M. M. (2017a). Perinatal Ciclosporin A Exposure Elicits Sex-Related Cardiac Dysfunction and Inflammation in the Rat Progeny. Toxicol. Lett. 281, 35-43. doi:10.1016/j.toxlet.2017.09.002

El-Bassossy, H. M., Banjar, Z. M., and El-Mas, M. M. (2017b). The Inflammatory State Provokes Sexual Dimorphism in Left Ventricular and Electrocardiographic Effects of Chronic Cyclosporine in Rats. Sci. Rep. 7, 42457. doi: $10.1038 /$ srep 42457

El-Bassossy, H. M., El-Maraghy, N. N., El-Fayoumi, H. M., and Watson, M. L. (2009). Haem Oxygenase-1 Induction Protects against Tumour Necrosis Factor Alpha Impairment of Endothelial-dependent Relaxation in Rat Isolated Pulmonary Artery. Br. J. Pharmacol. 158, 1527-1535. doi:10.1111/j.14765381.2009.00419.x

El-Bassossy, H. M., El-Moselhy, M. A., and Mahmoud, M. F. (2011). Pentoxifylline Alleviates Vascular Impairment in Insulin Resistance via TNF- $\alpha$ Inhibition. 
Naunyn Schmiedebergs Arch. Pharmacol. 384, 277-285. doi:10.1007/s00210011-0669-z

El-Bassossy, H. M., Elberry, A. A., Ghareib, S. A., Azhar, A., Banjar, Z. M., and Watson, M. L. (2016). Cardioprotection by 6-gingerol in Diabetic Rats. Biochem. Biophys. Res. Commun. 477, 908-914. doi:10.1016/j.bbrc.2016.06.157

El-Bassossy, H. M., Mahmoud, M. F., and Eid, B. G. (2018a). The Vasodilatory Effect of Allopurinol Mediates its Antihypertensive Effect: Effects on Calcium Movement and Cardiac Hemodynamics. Biomed. Pharmacother. 100, 381-387. doi:10.1016/j.biopha.2018.02.033

El-Bassossy, H. M., Neamatallah, T., Balamash, K. S., Abushareb, A. T., and Watson, M. L. (2018b). Arginase Overexpression and NADPH Oxidase Stimulation Underlie Impaired Vasodilation Induced by Advanced Glycation End Products. Biochem. Biophys. Res. Commun. 499, 992-997. doi:10.1016/j.bbrc.2018.04.036

El-Bassossy, H. M., and Watson, M. L. (2015). Xanthine Oxidase Inhibition Alleviates the Cardiac Complications of Insulin Resistance: Effect on Low Grade Inflammation and the Angiotensin System. J. Transl Med. 13, 82-11. doi:10.1186/s12967-015-0445-9

Engin, K. N. (2009). Alpha-tocopherol: Looking beyond an Antioxidant. Mol. Vis. $15,855-860$

Fahim, A. T., Abd-el Fattah, A. A., Agha, A. M., and Gad, M. Z. (1995). Effect of Pumpkin-Seed Oil on the Level of Free Radical Scavengers Induced during Adjuvant-Arthritis in Rats. Pharmacol. Res. 31, 73-79. doi:10.1016/10436618(95)80051-4

Guo, Y., Luo, J., Tan, S., Otieno, B. O., and Zhang, Z. (2013). The Applications of Vitamin E TPGS in Drug Delivery. Eur. J. Pharm. Sci. 49, 175-186. doi:10.1016/ j.ejps.2013.02.006

Hassan, N. A., Bassossy, H. M. E., Fahmy, A., and Mahmoud, M. F. (2018). Limonin Alleviates Macro- and Micro-vascular Complications of Metabolic Syndrome in Rats: A Comparative Study with Azelnidipine. Phytomedicine 43, 92-102. doi:10.1016/j.phymed.2018.03.044

Heitzer, T., Schlinzig, T., Krohn, K., Meinertz, T., and Münzel, T. (2001). Endothelial Dysfunction, Oxidative Stress, and Risk of Cardiovascular Events in Patients with Coronary Artery Disease. Circulation 104, 2673-2678. doi:10.1161/hc4601.099485

Inanır, M., Gunes, Y., Sincer, I., and Erdal, E. (2020). Evaluation of Electrocardiographic Ventricular Depolarization and Repolarization Variables in Type 1 Diabetes Mellitus. Arq Bras Cardiol. 114, 275-280. doi:10.36660/abc.20180343

Jagtap, S., Meganathan, K., Wagh, V., Winkler, J., Hescheler, J., and Sachinidis, A. (2009). Chemoprotective Mechanism of the Natural Compounds, Epigallocatechin-3-O-Gallate, Quercetin and Curcumin against Cancer and Cardiovascular Diseases. Curr. Med. Chem. 16, 1451-1462. doi:10.2174/ 092986709787909578

Kaur, J. (2014). A Comprehensive Review on Metabolic Syndrome. Cardiol. Res. Pract. 2014, 943162. doi:10.1155/2014/943162

Kumada, M., Kihara, S., Sumitsuji, S., Kawamoto, T., Matsumoto, S., Ouchi, N., et al. (2003). Association of Hypoadiponectinemia with Coronary Artery Disease in Men. Arterioscler Thromb. Vasc. Biol. 23, 85-89. doi:10.1161/ 01.atv. 0000048856.22331 .50

Mahmoud, M. F., El-Nagar, M., and El-Bassossy, H. M. (2012). Antiinflammatory Effect of Atorvastatin on Vascular Reactivity and Insulin Resistance in Fructose Fed Rats. Arch. Pharm. Res. 35, 155-162. doi:10.1007/s12272-012-0117-8

Mahmoud, M. F., Hassan, N. A., El Bassossy, H. M., and Fahmy, A. (2013). Quercetin Protects against Diabetes-Induced Exaggerated Vasoconstriction in Rats: Effect on Low Grade Inflammation. PloS one 8, e63784. doi:10.1371/ journal.pone.0063784

Murakami, A., Ashida, H., and Terao, J. (2008). Multitargeted Cancer Prevention by Quercetin. Cancer Lett. 269, 315-325. doi:10.1016/ j.canlet.2008.03.046
Olijhoek, J. K., van der Graaf, Y., Banga, J. D., Algra, A., Rabelink, T. J., Visseren, F. L., et al. (2004). The Metabolic Syndrome Is Associated with Advanced Vascular Damage in Patients with Coronary Heart Disease, Stroke, Peripheral Arterial Disease or Abdominal Aortic Aneurysm. Eur. Heart J. 25, 342-348. doi:10.1016/j.ehj.2003.12.007

Oliver, J. J., and Webb, D. J. (2003). Noninvasive Assessment of Arterial Stiffness and Risk of Atherosclerotic Events. Arterioscler Thromb. Vasc. Biol. 23, 554-566. doi:10.1161/01.ATV.0000060460.52916.D6

Pittas, A. G., Joseph, N. A., and Greenberg, A. S. (2004). Adipocytokines and Insulin Resistance. J. Clin. Endocrinol. Metab. 89, 447-452. doi:10.1210/jc.2003031005

Rivera, L., Morón, R., Sánchez, M., Zarzuelo, A., and Galisteo, M. (2008). Quercetin Ameliorates Metabolic Syndrome and Improves the Inflammatory Status in Obese Zucker Rats. Obesity (Silver Spring) 16, 2081-2087. doi:10.1038/ oby.2008.315

Sarazan, R. D., Kroehle, J. P., and Main, B. W. (2012). Left Ventricular Pressure, Contractility and $\mathrm{dP} / \mathrm{dt}(\max )$ in Nonclinical Drug Safety Assessment Studies. J. Pharmacol. Toxicol. Methods 66, 71-78. doi:10.1016/j.vascn.2012.05.009

Shen, Y., Croft, K. D., Hodgson, J. M., Kyle, R., Lee, I. L., Wang, Y., et al. (2012). Quercetin and its Metabolites Improve Vessel Function by Inducing eNOS Activity via Phosphorylation of AMPK. Biochem. Pharmacol. 84, 1036-1044. doi:10.1016/j.bcp.2012.07.016

Taghipour, Y. D., Hajialyani, M., Naseri, R., Hesari, M., Mohammadi, P., Stefanucci, A., et al. (2019). Nanoformulations of Natural Products for Management of Metabolic Syndrome. Int. J. Nanomedicine 14, 5303-5321. doi:10.2147/IJN.S213831

Visioli, F. (2011). Nutritional Support in the Pharmacological Treatment of Metabolic Syndrome. Eur. J. Pharmacol. 668 (Suppl. 1), S43-S49. doi:10.1016/j.ejphar.2011.05.083

Wein, S., Behm, N., Petersen, R. K., Kristiansen, K., and Wolffram, S. (2010), Quercetin Enhances Adiponectin Secretion by a PPAR-Gamma Independent Mechanism. Eur. J. Pharm. Sci. 41, 16-22. doi:10.1016/j.ejps.2010.05.004

Yang, C., Wu, T., Qi, Y., and Zhang, Z. (2018). Recent Advances in the Application of Vitamin E TPGS for Drug Delivery. Theranostics 8, 464-485. doi:10.7150/ thno. 22711

Zhang, J., Zhao, L., Cheng, Q., Ji, B., Yang, M., Sanidad, K. Z., et al. (2018). Structurally Different Flavonoid Subclasses Attenuate High-Fat and HighFructose Diet Induced Metabolic Syndrome in Rats. J. Agric. Food Chem. 66, 12412-12420. doi:10.1021/acs.jafc.8b03574

Zhang, Z., Tan, S., and Feng, S. S. (2012). Vitamin E TPGS as a Molecular Biomaterial for Drug Delivery. Biomaterials 33, 4889-4906. doi:10.1016/ j.biomaterials.2012.03.046

Conflict of Interest: The authors declare that the research was conducted in the absence of any commercial or financial relationships that could be construed as a potential conflict of interest.

Publisher's Note: All claims expressed in this article are solely those of the authors and do not necessarily represent those of their affiliated organizations, or those of the publisher, the editors and the reviewers. Any product that may be evaluated in this article, or claim that may be made by its manufacturer, is not guaranteed or endorsed by the publisher.

Copyright () 2021 Ahmed, Hassan, Azhar, El-Mas and El-Bassossy. This is an openaccess article distributed under the terms of the Creative Commons Attribution License (CC BY). The use, distribution or reproduction in other forums is permitted, provided the original author(s) and the copyright owner(s) are credited and that the original publication in this journal is cited, in accordance with accepted academic practice. No use, distribution or reproduction is permitted which does not comply with these terms. 